\title{
OBESIDADE, PERFIL LIPÍDICO E HÁBITOS ALIMENTARES DE ESCOLARES: COMPARAÇÃO ENTRE MUNICÍPIOS DE DOIS ESTADOS DA REGIÃO SUL DO BRASIL
}

\section{Cassiano Macari}

Mestre em Promoção da Saúde, pelo programa de Pós-graduação em Promoção da Saúde, da Universidade de Santa Cruz do Sul, Santa Cruz do Sul (UNISC), Brasil.

\section{Andréia Rosane de Moura Valim}

Doutora em Biologia Celular e Molecular (UFRGS), Docente do Programa de Pós-graduação - Mestrado em Promoção da Saúde e do Departamento de Biologia e Farmácia, da Universidade de Santa Cruz do Sul (UNISC), Brasil.

\section{Clodoaldo Antônio De Sá}

Doutor em Ciência do Movimento Humano (UFSM). Docente do Programa de Pós-graduação em Ciências da Saúde (Mestrado e Doutorado), da Universidade Comunitária da Região de Chapecó (Unochapecó), Chapecó (SC), Brasil.

\section{Priscila Tatiana da Silva}

Graduada em Educação Física (UNISC). Mestranda em Promoção da Saúde, no Programa de Pós-graduação em Promoção da Saúde, da Universidade de Santa Cruz do Sul (UNISC), Brasil.

\section{Cláudia Daniela Barbian}

Graduada em Educação Física (UNISC). Mestranda em Promoção da Saúde, no Programa de Pós-graduação em Promoção da Saúde, da Universidade de Santa Cruz do Sul (UNISC), Brasil.

\section{Miria Suzana Burgos}

Doutora em Ciências de La Educación (UPSA). Docente do Departamento de Educação Física e Saúde e do Programa de Pós-graduação - Mestrado em Promoção da Saúde, da Universidade de Santa Cruz do Sul (UNISC), Brasil

\section{Lia Gonçalves Possuelo}

Doutora em Ciências Biológicas - Bioquímica (UFRGS). Docente adjunto da UNISC e do Programa de Pós-graduação Mestrado em Promoção da Saúde, da Universidade de Santa Cruz do Sul (UNISC), Brasil.

E-mail: liapossuelo@unisc.br
RESUMO: O objetivo foi comparar o perfil nutricional, lipídico e hábitos alimentares de escolares de dois municípios do sul do Brasil. O estudo transversal avaliou indicadores de obesidade, perfil lipídico e hábitos alimentares de escolares de 7-17 anos, de Santa Cruz do Sul-RS e Chapecó-SC. Observou-se diferença $(p<0,05)$ no sobrepeso/obesidade (37,0\% Chapecó vs 29,2\% Santa Cruz do Sul) e circunferência da cintura elevada (34,8\% Chapecó vs 21,2\% Santa Cruz do Sul); triglicerídeos $(41,4 \%)$ e HDL-C $(23,5 \%)$ foram mais elevados em Chapecó $(p<0,001)$ e colesterol total $(56,1 \%)$, LDL-C $(36,4 \%)$ e glicemia $(8,7 \%)$ em Santa Cruz do Sul $(p<0,05)$. Chapecó apresenta maior consumo de frutas (16,8\% infrequente) e doces ( $41,3 \%$ frequente) e Santa Cruz do Sul de feijão/arroz (57,0\% diário), refrigerantes (24,4\% diário) e salgadinhos (24,0\% diário) $\mathrm{p}<0,05$. Conclui-se que houve diferenças no perfil nutricional, lipídico e hábitos alimentares entre dois municípios, demonstrando que indicadores de saúde podem variar em diferentes regiões.

PALAVRAS-CHAVE: Composição corporal; Criança; Adolescente; Comportamento alimentar; Lipídeos.

\section{OBESITY, LIPID PROFILE AND FEEDING HABITS IN SCHOOL CHILDREN: COMPARING MUNICIPALITIES IN T WO BRAZILIAN SOUTHERN STATES}

ABSTRACT: Nutritional, lipid and feeding habits of school children in two municipalities in South Brazil are compared. Transversal study analyzed indicators of obesity, lipid profile and feeding habits of school children, aged between 7 and 17 years, in Santa Cruz do Sul RS Brazil, and Chapecó SC Brazil. Difference $(\mathrm{p}<0.05)$ in overweight/obesity $(37.0 \%$ Chapecó vs 29.2\% Santa Cruz do Sul) and high waist circumference (34.8\% Chapecó vs 21.2\% Santa Cruz do Sul); triglycerides (41.4\%) and HDL-C $(23.5 \%)$ showed higher rates in Chapecó $(\mathrm{p}<0.001)$; total cholesterol (56.1\%), LDL-C (36.4\%) and glycaemia (8.7\%) were higher in Santa Cruz do Sul $(\mathrm{p}<0.05)$. Chapecó had a high input of fruit (16.8\% infrequent) and sweets (41.3\% frequent) contrasting with beans/rice (57.0\% daily), soft drinks (24.4\% daily) and snacks $(24.0 \%$ daily) in Santa Cruz do Sul $(\mathrm{p}<0.05)$. Results show that differences in nutritional profile existed between the two municipalities and, consequently, health indicators vary within different regions.

KEY WORDS: Body composition; Child; Adolescent; Feeding behavior; Lipids. 


\section{INTRODUÇÃO}

A população brasileira teve um aumento de poder econômico e tecnológico, e apesar dos inegáveis benefícios que essas mudanças promoveram, induziu-se uma mudança nos hábitos alimentares, com aumento na disponibilidade e consumo de alimentos processados semiprontos de fácil preparo (IBGE, 2010). Alimentos de baixo valor nutricional, ricos em açúcares, gorduras e sal, pobres em fibras e micronutrientes estão sendo consumidos de forma crescente, sendo este comportamento alimentar associado a outros fatores de risco para a saúde, como o sedentarismo resultante da redução da prática de atividades físicas (MUSAIGER; FALAN, 2014).

Hábitos alimentares são formados por influência de diversos fatores, entre eles culturais, econômicos, psicológicos e fisiológicos. Estes hábitos são desenvolvidos na infância e consolidados no início da fase adulta, tendo a família grande influência na adoção de comportamentos alimentares. Em relação a esses aspectos nutricionais, a população está apresentando uma nova forma de se alimentar, denominada de transição nutricional. Este período se caracteriza pela redução da prevalência dos déficits nutricionais, como a desnutrição e o baixo peso, e pela ascensão do excesso de peso na população (WANG et al., 2017). Este último ocorre de forma indistinta, afetando populações adultas e pediátricas, de todas as classes sociais, sendo conhecido como importante problema de saúde pública (MUSAIGER; FALAN, 2014).

Segundo dados do Instituto Brasileiro de Pesquisa e Estatística (IBGE), quase metade (49\%) da população brasileira está com excesso de peso e destes, $14,8 \%$ são obesos. No que se refere à obesidade infantil, a Organização Mundial de Saúde (OMS) estima que a prevalência mundial tenha aumentado em torno de $10 \mathrm{a}$ $40 \%$, na maioria dos países. No Brasil, esse índice subiu $240 \%$ nas últimas duas décadas, variando de região para região, sendo mais prevalente nas regiões Sul e Sudeste, com índices variando de 32 a 40\% (IBGE, 2010). Essas diferenças nos índices de saúde, entre diferentes regiões, podem ser decorrentes do contexto socioeconômico, comportamental e ambiental destes locais. Desta forma, torna-se importante obter uma visão atual do contexto de saúde, para que futuras estratégias sejam efetuadas para minimizar esse quadro, principalmente nos locais onde as taxas de risco à saúde são mais expressivas (DREWNOWSKI et al., 2014).

Diante deste contexto, o monitoramento da situação de saúde destas populações, com ações que permitam comparações ao longo do tempo e o reconhecimento de fatores associados, são estratégias extremamente úteis para a elaboração de políticas públicas de saúde e programas que atuem na prevenção e controle desta nova realidade. Sob este contexto, o objetivo do presente estudo foi comparar o perfil nutricional, lipídico e hábitos alimentares de escolares de dois municípios do sul do Brasil.

\section{MÉTODO}

Foi realizado um estudo transversal nos municípios de Santa Cruz do Sul (RS) e Chapecó (SC), no período de maio a novembro de 2013 e 2014, respectivamente. Santa Cruz do Sul é uma cidade localizada na encosta inferior do Estado do Rio Grande do Sul, com uma população de aproximadamente 120 mil habitantes e índice de desenvolvimento humano (IDH) de 0,773 ; de colonização germânica, sendo esta a etnia predominante na população atual, no qual a plantação e beneficiamento do fumo é a sua principal fonte econômica (SANTA CRUZ DO SUL, 2017). Chapecó está localizada no extremo oeste catarinense e possui uma população aproximada de 190 mil habitantes e um IDH de 0,790 . Conhecida como a capital brasileira das agroindústrias, tem neste setor, sua principal fonte de emprego e renda (CHAPECÓ, 2017).

O projeto de pesquisa foi aprovado pelo Comitê de Ética e Pesquisa da Universidade de Santa Cruz do SulUNISC, sob o parecer número 469.950 e está de acordo com as normas da resolução 466/2012 do Conselho Nacional de Saúde sobre pesquisa, envolvendo seres humanos.

Os escolares foram selecionados aleatoriamente, a partir da localização geográfica das escolas, abrangendo assim todos os quadrantes (norte, sul, leste, oeste e região central) das cidades envolvidas. Os escolares das escolas 
selecionadas foram convidados a participar da pesquisa, levando para casa os instrumentos de coleta de dados (questionário sobre a condição socioeconômica e dados pessoais), a serem respondidos pelos responsáveis, bem como o termo de consentimento livre e esclarecido (TCLE), para assinatura dos pais ou responsáveis. O recolhimento do material enviado às residências, assim como a realização das coletas de dados antropométricos e de sangue foram efetuadas no ambiente escolar, em data previamente definida, junto à escola e em local destinado para este fim.

Foram adotados como critérios de inclusão para a participação no estudo, estar na faixa etária entre sete e 17 anos, preenchimento dos instrumentos de coleta de dados e um jejum de 12 horas, para obtenção de amostras de sangue total. Foram excluídos do estudo, aqueles escolares com instrumento de coleta com dados incompletos ou que se negaram a realizar os procedimentos de coleta de sangue e/ou de dados antropométricos.

Para definição de obesidade e risco de obesidade foi usado o índice de massa corporal (IMC). Tal índice é obtido pela divisão dos valores de massa corporal em quilogramas, pelos valores de estatura em metros quadrados. O critério estabelecido para a obesidade, de acordo com o gênero e a idade é o de IMC $\geq$ percentil 95 , e sobrepeso é o de IMC entre os percentis 85 a 95 (CDC/ NCHS, 2002). Para medida da massa corporal foi utilizada balança digital (G-Tech Balg4FB), com capacidade máxima de $180 \mathrm{~kg}$ e precisão de 100 gramas, enquanto que para a estatura foi usado um estadiômetro em alumínio (Sanny). Posteriormente, o IMC foi classificado de acordo com o protocolo de Conde e Monteiro (2006), em baixo peso/ pesonormal e sobrepeso/obesidade.

Foi utilizada uma fita métrica inelástica para medidas de circunferência de cintura (CC), mensurada no ponto médio entre a crista ilíaca e a face externa da última costela, sendo posteriormente classificados de acordo com os critérios estabelecidos por Taylor et al. (2000), que consideram a circunferência normal (percentil $\leq 80$ ) e elevada (percentil $>80$ ), de acordo com o sexo e a idade.

Para avaliação do percentual de gordura (\%G), foi utilizado o somatório das dobras cutâneas tricipital e subescapular, obtidas pela medição com compasso de Lange. Para o cálculo do \% G, foi utilizada a equação de
Slaugtheret al. (1988); sendo posteriormente classificado de acordo com de Lohman (1987), que preconiza de $10 \mathrm{a}$ $15 \%$ ideal para os meninos e de 16 a $23 \%$ para as meninas.

Paraaavaliaçãodosindicadores socioeconômicos, foi utilizado um questionário, de acordo com o critério da Associação Brasileira de Empresas de Pesquisa (ABEP), que tem como função estimar o poder de compra das pessoas e famílias urbanas, sendo os grupos populacionais divididos em classes sociais: A (A1 + A2), B (B1 + B2), C, D e E (ABEP, 2011). Para o item instrução de chefe da família foram consideradas quatro categorias: analfabeto/ primário incompleto, ensino fundamental completo/ incompleto, ensino médio completo/incompleto e ensino superior.

A investigação dos hábitos alimentares foi realizada por meio do questionário adaptado de Barros e Nahas (2003). Posteriormente, a frequência de consumo destes alimentos foi classificada da seguinte forma: infrequente quando não consumidos ou consumidos uma vez por semana, frequentes quando consumidos de duas a seis vezes por semana, diários, quando consumidos de uma a três vezes ao dia.

Para avaliar os marcadores de perfil lipídico, o escolar foi submetido a uma coleta sanguínea, após um período de 12 horas de jejum, sem ter realizado exercícios físicos. Para tanto, foram coletados cerca de $10 \mathrm{~mL}$ de sangue da veia braquial. Os parâmetros bioquímicos (glicemia, colesterol total e triglicerídeos) foram avaliados, utilizando amostras de soro em equipamento automatizado MiuraOne (ISE, Roma, Itália), utilizando-se kit comercial de Dia Sys (Dia SysDiagnostic Systems, Alemanha). Os pontos de corte adotados para glicemia, colesterol total e suas frações e triglicerídeos foram recomendados na I Diretriz de Prevenção da Aterosclerose na Infância e Adolescência da Sociedade Brasileira de Cardiologia que prevê: glicemia aumentada $\geq 100 \mathrm{mg} / \mathrm{dL}$, colesterol total aumentado $\geq 170 \mathrm{mg} / \mathrm{dL}$, HDL-C $\leq 45 \mathrm{mg} / \mathrm{dL}, \mathrm{LDL} \geq 130 \mathrm{mg} / \mathrm{dL}$ e triglicerídeos aumentado para valores $\geq 130 \mathrm{mg} / \mathrm{dL}$ (SBC, 2013).

Os dados foram tabulados e analisados no Programa SPSS (v 20.0). Foi realizada análise descritiva para verificação dos padrões de distribuição e tendências das principais variáveis (números absolutos, frequências e médias). Foram empregados testes Qui-quadrado ( $\left.\mathrm{c}^{2}\right)$ para diferenças de proporção e testes $t$ de Student, para diferenças entre médias. Os valores estão expressos em 
frequências, médias e desvio-padrão, considerando um nível de significância de 5\% ( $\mathrm{p}<0,05)$. A magnitude de associação entre as variáveis foi expressa em Razão de prevalência (R-P) e respectivos intervalos de confiança de 95\% (IC95\%).

\section{RESULTADOS}

Foram avaliadas 658 crianças e adolescentes de sete a 17 anos, sendo $353(53,65 \%)$ da cidade de Santa
Cruz do Sul e 305 (46,35\%) da cidade de Chapecó. Na Tabela 1 é apresentada descrição das variáveis analisadas como gênero, idade, nível socioeconômico, grau de instrução do chefe da família. A média de idade dos escolares foi 10,89 $\pm 2,32$ anos, sendo que $53,3 \%$ eram do sexo feminino, $92,0 \%$ das famílias pertence às classes socioeconômicas B e C e $61,2 \%$ dos chefes de família possuíam o ensino fundamental, como instrução educacional. Observou-se que no município de Chapecó, o grau de instrução dos chefes de família é superior ao observado em Santa Cruz do Sul $(\mathrm{p}=0,002)$.

Tabela 1. Distribuição da amostra em relação ao gênero, idade, nível socioeconômico, grau de instrução do chefe da família e classificação de IMC, estratificado por cidade

\begin{tabular}{|c|c|c|c|c|}
\hline Variáveis & $\begin{array}{c}\text { SCS }(\mathrm{n}=353) \\
\mathrm{n}(\%)\end{array}$ & $\begin{array}{c}\mathrm{CCO}(\mathrm{n}=305) \\
\mathrm{n}(\%)\end{array}$ & $p$ & $\begin{array}{l}\text { Total } \\
\text { n (\%) }\end{array}$ \\
\hline Sexo & & & 0,876 & \\
\hline Masculino & $166(47,0)$ & $141(46,2)$ & & $307(46,7)$ \\
\hline Feminino & $187(53,0)$ & $164(53,8)$ & & $351(53,3)$ \\
\hline Faixa etária & & & 0,206 & \\
\hline 7 a 9 anos & $112(31,7)$ & $83(27,2)$ & & $195(29,6)$ \\
\hline 10 a 17 anos & $241(68,3)$ & $222(72,8)$ & & $463(70,4)$ \\
\hline Classificação econômica & & & 0,064 & \\
\hline A & $1(0,3)$ & $10(3,3)$ & & $11(1,7)$ \\
\hline B & $101(28,6)$ & $91(29,8)$ & & $192(29,2)$ \\
\hline $\mathrm{C}$ & $228(64,6)$ & $185(60,7)$ & & $413(62,8)$ \\
\hline $\mathrm{D}$ & $22(6,2)$ & $18(5,9)$ & & $40(6,0)$ \\
\hline $\mathrm{E}$ & $1(0,3)$ & $1(0,3)$ & & $2(0,3)$ \\
\hline Grau de instrução chefe da Família & & & 0,002 & \\
\hline Analfabeto/Primário Incompleto até 4série & $81(22,9)$ & $62(20.9)$ & & $143(21,9)$ \\
\hline Ensino Fundamental completo/incompleto & $213(60,3)$ & $186(62,6)$ & & $399(61,2)$ \\
\hline Ensino Médio completo/incompleto & $46(13,0)$ & $20(6,7)$ & & $66(10,1)$ \\
\hline Ensino Superior & $13(3,7)$ & $26(8,8)$ & & $39(6)$ \\
\hline
\end{tabular}

SCS: Santa Cruz do Sul; CCO: Chapecó; IMC: Índice de Massa Corporal

No que se refere aos indicadores antropométricos (Tabela 2), a prevalência de sobrepeso/obesidade $(37,0 \%$ Chapecó vs 29,2\% Santa Cruz do Sul) e CC elevada (34,8\% Chapecó vs 21,2\% Santa Cruz do Sul) foram maiores em
Chapecó $(p<0,05)$. Não houve diferença estatística em relação ao \%G, entretanto a frequência de \% G moderado/ alto/muito alto foi maior em Santa Cruz do Sul (46,5\%). 
Tabela 2. Indicadores de sobrepeso e obesidade, valores médios (DP), frequências e razão de prevalência (RP) das crianças dos dois municípios

\begin{tabular}{|c|c|c|c|c|c|}
\hline Variáveis & $\begin{array}{c}\text { SCS }(\mathbf{n}=353) \\
n(\%)\end{array}$ & $\begin{array}{c}\operatorname{CCO}(n=305) \\
n(\%)\end{array}$ & $p$ & RP (IC95\%) & $\begin{array}{l}\text { Total } \\
\text { n (\%) }\end{array}$ \\
\hline IMC & & & 0,032 & $1,42(1,03-1,98)$ & \\
\hline Peso Normal & $250(70,8)$ & $192(63,0)$ & & & $442(67,22)$ \\
\hline Sobrepeso/Obesidade & $103(29,2)$ & $113(37,0)^{*}$ & & & $216(32,8)$ \\
\hline$\% \mathrm{G}$ & & & 0,059 & $0,74(0,54-1,01)$ & \\
\hline Baixo/Ótimo & $189(53,5)$ & $185(60,9)$ & & & $374(56,9)$ \\
\hline Mod./Alto/Muito alto & $164(46,5)$ & $119(39,1)$ & & & $283(43,1)$ \\
\hline $\mathrm{CC}$ & $65,3( \pm 10,54)$ & $65,57( \pm 10,16)$ & $<0,001$ & $1,97(1,39-2,79)$ & \\
\hline Normal & $278(78,8)$ & $199(65,2)$ & & & $477(72,5)$ \\
\hline Elevado & $75(21,2)$ & $106(34,8) *$ & & & $181(27,5)$ \\
\hline
\end{tabular}

SCS: Santa Cruz do Sul; CCO: Chapecó; IMC: Índice de Massa Corporal; DP: Desvio-padrão; \%G: Percentual de gordura; CC: Circunferência da cintura; RP: Razão de prevalência. * Diferença significativa

Ao observar os resultados referentes ao perfil bioquímico (Tabela 3), verificou-se que as médias de triglicerídeos e HDL-C foram maiores em Chapecó, e as médias de colesterol total, LDL-C e glicemia mais expressivas em Santa Cruz do Sul. Em relação aos níveis de triglicerídeos, 41,4\% dos escolares de Chapecó apresentaram valores alterados $(\mathrm{p}<0,001)$, enquanto que o HDL-C se mostrou alterado em $23,5 \%$ dos escolares $(p<0,001) .56,1 \%, 36,4 \%$ e 8,7\% dos escolares de Santa Cruz do Sul apresentaram níveis alterados de colesterol total, LDL-C e glicemia $(\mathrm{p}<0,05)$.

Tabela 3. Características do perfil lipídico dos escolares, estratificado por cidade

\begin{tabular}{|c|c|c|c|c|c|}
\hline Variáveis & $\begin{array}{c}\text { SCS } \\
\text { n (\%) }\end{array}$ & $\begin{array}{l}\text { CCO } \\
\text { n (\%) }\end{array}$ & $p$ & RP(IC95\%) & $\begin{array}{l}\text { Total } \\
\text { n (\%) }\end{array}$ \\
\hline TRIGLICERÍDEOS* & & & $<0,001$ & $4,94(3,1-7,7)$ & \\
\hline Desejável & $301(87,5)$ & $99(58,6)$ & & & $400(78,0)$ \\
\hline Alterado & $43(12,5)$ & $70(41,4)^{* *}$ & & & $113(22,0)$ \\
\hline COLESTEROL TOTAL* & & & $<0,001$ & $0,46(0,31-0,69)$ & \\
\hline Desejável & $119(43,9)$ & $104(62,7)$ & & & $223(51,0)$ \\
\hline Alterado & $152(56,1)^{* *}$ & $62(37,3)$ & & & $214(49,0)$ \\
\hline GLICOSE* & & & 0,004 & $0,2(0,06-0,67)$ & \\
\hline Desejável & $314(91,3)$ & $154(98,3)$ & & & $468(93,4)$ \\
\hline Alterado & $30(8,7)^{* *}$ & $3(1,9)$ & & & $33(6,6)$ \\
\hline HDL-C* & & & 0,001 & $0,46(0,29-0,74)$ & \\
\hline Desejável & $292(86,4)$ & $124(74,7)$ & & & $416(82,5)$ \\
\hline Alterado & $46(13,6)$ & $42(23,5)^{* *}$ & & & $88(17,5)$ \\
\hline LDL-C* & & & 0,026 & $0,62(0,41-0,94)$ & \\
\hline Desejável & $213(63,6)$ & $120(73,6)$ & & & $333(66,9)$ \\
\hline Alterado & $122(36,4)^{* *}$ & $43(26,4)$ & & & $165(33,1)$ \\
\hline
\end{tabular}

*Triglicerídeos: $\geq 100 \mathrm{mg} / \mathrm{dL}$; Colesterol Total: $\geq 150 \mathrm{mg} / \mathrm{dL}$; Glicose: $\geq 99 \mathrm{mg} / \mathrm{dL} ;$ HDL-C: $\leq 45 \mathrm{mg} / \mathrm{dL}$; LDL-C: $\geq 100 \mathrm{mg} / \mathrm{dL} ;$ ** Diferenças significativas; RP:Razão de prevalência; SCS:Santa Cruz do Sul; CCO:Chapecó 
De modo geral, os escolares das duas cidades analisadas possuem um padrão alimentar semelhante (Tabela 4), com baixo consumo diário de frutas $(36,0 \%$ Santa Cruz do Sul vs 39,2\% Chapecó), saladas e legumes (30,3\% Santa Cruz do Sul vs 32,2\% Chapecó) somada ao consumo frequente de doces (31,1\% Santa Cruz do Sul vs 41,3\% Chapecó), salgadinhos fritos (34,6\% Santa Cruz do Sul vs 38,4\% Chapecó), e refrigerantes (41,6\%
Santa Cruz do Sul vs 39,4\% Chapecó). Houve diferenças entre as duas cidades analisadas $(p<0,05)$ em que Chapecó apresentou maior consumo de frutas (16,8\% infrequente) e doces ( $41,3 \%$ frequente) e Santa Cruz do Sul apresentou consumo mais elevado de feijão e arroz ( $57 \%$ diário), refrigerantes (24,4\% diário) e salgadinhos fritos (24,0\% diário)

Tabela 4. Distribuição das frequências dos alimentos considerados saudáveis e não saudáveis, estratificados por cidade

\begin{tabular}{|c|c|c|c|}
\hline Variáveis & $\begin{array}{l}\text { SCS }(\mathrm{n}=353) \\
\mathrm{n}(\%)\end{array}$ & $\begin{array}{c}\operatorname{CCO}(\mathbf{n}=305) \\
\mathbf{n}(\%)\end{array}$ & $\mathbf{p}$ \\
\hline \multicolumn{4}{|c|}{ Suco de frutas } \\
\hline Infrequente & $161(45,6)$ & $137(45,0)$ & \\
\hline Frequente & $119(33,7)$ & $105(34,5)$ & 0,74 \\
\hline Diário & $63(20,7)$ & $63(20,5)$ & \\
\hline \multicolumn{4}{|l|}{ Frutas } \\
\hline Infrequente & $90(25,5)^{*}$ & $51(16,8)^{*}$ & \\
\hline Frequente & $136(38,5)$ & $134(44,0)$ & 0,02 \\
\hline Diário & $127(36,0)$ & $120(39,2)$ & \\
\hline \multicolumn{4}{|c|}{ Saladas e legumes } \\
\hline Infrequente & $135(38,3)$ & $91(29,8)$ & 0,06 \\
\hline Frequente & $111(31,3)$ & $116(38,0)$ & \\
\hline Diário & $107(30,3)$ & $98(32,2)$ & \\
\hline \multicolumn{4}{|l|}{ Batatas } \\
\hline Infrequente & $203(57,5)$ & $185(60,9)$ & 0,48 \\
\hline Frequente & $96(27,1)$ & $83(27,3)$ & \\
\hline Diário & $54(15,4)$ & $37(11,0)$ & \\
\hline \multicolumn{4}{|c|}{ Refrigerantes } \\
\hline Infrequente & $120(34,0)$ & $133(43,6)$ & 0,01 \\
\hline Frequente & $147(41,6)$ & $120(39,4)$ & \\
\hline Diário & $86(24,4)^{*}$ & $52(17,9)^{*}$ & \\
\hline \multicolumn{4}{|c|}{ Salgadinhos fritos } \\
\hline Infrequente & $146(41,4)$ & $144(47,2)$ & 0,00 \\
\hline Frequente & $122(34,6)$ & $117(38,4)$ & \\
\hline Diário & $85(24,0)^{*}$ & $44(14,4)^{*}$ & \\
\hline \multicolumn{4}{|c|}{$\begin{array}{l}\text { Pizzas/Lasanhas e outros alimentos com queijos e } \\
\text { molho branco }\end{array}$} \\
\hline Infrequente & $244(69,1)$ & $196(60,8)$ & 0,05 \\
\hline Frequente & $62(17,5)$ & $79(26,0)$ & \\
\hline
\end{tabular}




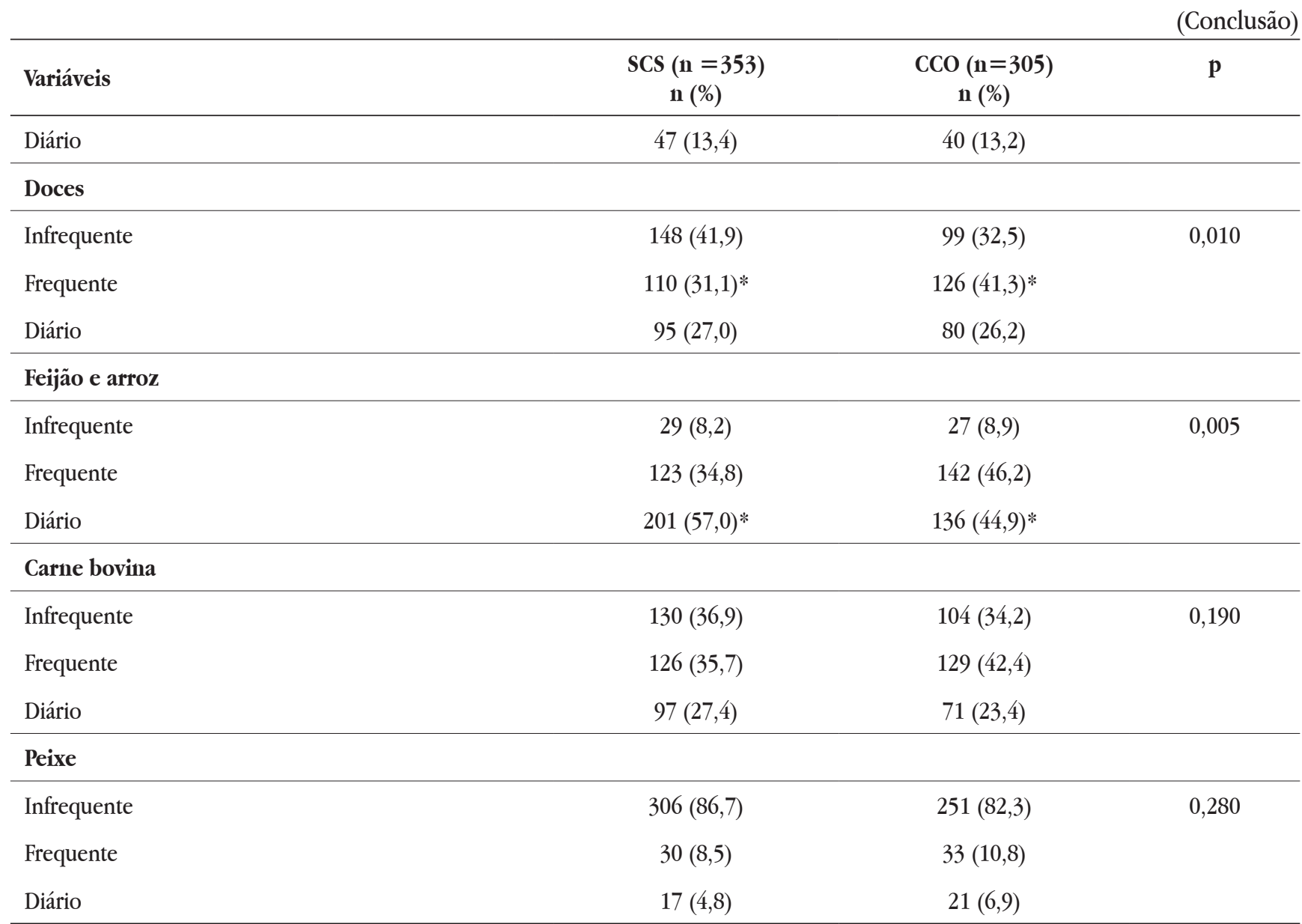

SCS: Santa Cruz do Sul; CCO: Chapecó. * Diferença significativa.

\section{DISCUSSÃO}

Quando comparados os dois municípios de nosso estudo, a prevalência de excesso de peso foi maior em Chapecó, demonstrando que indicadores de saúde podem variar conforme a região, sendo essa diferença percebida também em outros estudos nacionais e de outros países ocidentais, onde os índices de sobrepeso/ obesidade variam conforme a região ou países estudados. $\mathrm{Na}$ cidade de Nandes-SP, um estudo com 402 crianças e adolescentes, a prevalência de sobrepeso/obesidade foi 30,59\% (CABRERA et al., 2014).

Na China, achados demonstraram, em 2014, uma taxa de prevalência de sobrepeso/obesidade de 19,4\% entre escolares de sete a 18 anos, sendo que a tendência de aumento de sobrepeso, durante o período de 1985 a 2014, aumentou continuamente, com a taxa anual variando entre $0,27 \%$ a $0,63 \%$ e a obesidade variando entre $0,10 \%$ e $0,58 \%$ (WANG et al., 2017). Na Polônia, um estudo com 160 escolares, entre dez e 12 anos, das cidades de Skawina e Crácovia, constataram-se índices ainda mais elevados, com a prevalência de sobrepeso/ obesidade, alcançando 33,2\% dos adolescentes avaliados (PYSZ et al., 2014).

No Estado de Santa Catarina, um estudo envolvendo oito municípios-polo, incluindo as cidades de Chapecó, Joaçaba, Blumenau, Jaraguá do Sul, Lages, Criciúma, Florianópolis e Joinville, avaliou 4.964 escolares, com idades entre seis a dez anos, revelou resultados similares e apontou uma prevalência de 15,4\% de sobrepeso e $6,0 \%$ de obesidade. O estudo constatou também que hábitos comportamentais, como prática reduzida de esportes e horas de sono à noite, bem como mais tempo no computador associaram-se com sobrepeso e obesidade nos escolares avaliados (CORSO et al., 2012); em Santa Maria-RS, um estudo transversal realizado com adolescentes, de 14 a 18 anos, demonstrou que a prevalência de sobrepeso/obesidade nos escolares 
avaliados alcançou 23,8\% da amostra (CUREAU et al., 2014). Esses dados reforçam as estimativas de que a maior prevalência de sobrepeso/obesidade para a população infantojuvenil brasileira encontra-se nas regiões Sul e Sudeste, sendo que essas diferenças de perfil nutricional entre as regiões podem ser explicadas pelas características socioculturais e demográficas, onde estas regiões estão inseridas (BLOCH et al., 2016). Assim, as evidências apontadas confirmam a tendência crescente de excesso de peso em crianças e adolescentes, em idade escolar (PYSZ et al., 2014; WANG et al., 2017).

Em relação ao percentual de gordura corporal alterado, observou-se que ambos os municípios de nosso estudo apresentaram elevadas taxas (46,5\% Santa Cruz do Sul vs 39,1\% Chapecó), porém não se observou diferença significativa. No entanto, resultados diversificados têm sido apontados em outros estudos, indicando que a prevalência pode variar conforme o local estudado. Em São Caetano do Sul-SP, um estudo realizado com escolares, de nove a 12 anos, apontou prevalência de sobrepeso/obesidade em 51,8\% da amostra avaliada. Os fatores associados aos valores de IMC foram porcentagem de gordura corporal $(\beta=0,0216 ; \mathrm{p}<0,001)$ e tempo de tela $(\beta=0,0050 ; p=0,006)$, sendo que apenas a porcentagem de gordura corporal esteve associada como fator comum entre ambos os sexos (FERRARI et al., 2017).

Dados do estudo multinacional de obesidade infantil, realizado com 7.265 crianças, de nove a 11 anos de idade, em 12 países (Austrália, Brasil, Canadá, China, Colômbia, Finlândia, Índia, Quênia, Portugal, África do Sul, Reino Unido e Estados Unidos), também apontaram alta associação entre IMC e porcentagem de gordura corporal, sendo que em todos os casos, o maior nível de gordura corporal foi em crianças indianas e o menor em crianças de Portugal e do Quênia (KATZMARZYK et al., 2015).

Um estudo anterior realizado em Santa Cruz do Sul, com 1.666 escolares, com idades entre sete e 17 anos, demonstrou que 35,9\% dos escolares apresentavam porcentagem de gordura corporal acima de moderadamente alto. Além disso, observou-se que a presença de hipertensão arterial esteve associada com obesidade e capacidade respiratória; aspectos que reforçam uma ligação entre IMC e níveis pressóricos em escolares, apontando para a importância de se estabelecer um estilo de vida mais ativo e saudável na infância e adolescência (BURGOS et al., 2010).

No presente estudo, na comparação entre os municípios, os valores de CC se apresentam elevados em $21,2 \%$ e 34,8\% dos escolares de Santa Cruz do Sul e Chapecó, respectivamente. ACC, por ser uma variável que mensura a quantidade de gordura abdominal, se constitui como importante preditor de doenças cardiovasculares (SBC, 2013), o que demonstra a necessidade de avaliação, tendo em vista a diferença na prevalência em diferentes estudos. Na Coreia do Sul, estudo com escolares, de dez a 19 anos, demonstrou associação significativa entre comorbidades metabólicas e obesidades $(\mathrm{p}<0,05)$. Meninos apresentaram valores mais elevados de IMC, $\mathrm{CC}$ e PA. Entre as meninas constataram-se valores mais elevados de HDL-C e colesterol total $(\mathrm{p}<0,05)$. Ainda, crianças e adolescentes obesos apresentaram risco aumentado de apresentar PA elevada, dislipidemia, TG elevado, baixo HDL-C e comorbidades em comparação com indivíduos com peso normal (LIM et al., 2014).

Quanto ao perfil lipídico, os escolares, de ambos os municípios, apresentam padrões de dislipidemia, tendo a prevalência diferido entre eles. $\mathrm{Na}$ cidade de Chapecó, os escolares apresentaram taxas mais elevadas de triglicerídeos $(41,4 \%)$ e níveis abaixo do recomendável para o HDL (23,5\%), e em Santa Cruz do Sul taxas mais elevadas de colesterol total $(56,1 \%)$, LDL-C $(36,4 \%)$ e glicose $(8,7 \%)$. Prevalência maior de TG $(63,0 \%)$ e HDL-C $(44,1 \%)$ foram observadas no norte do México, em estudo com 451 crianças, de dois a dez anos, porém os níveis alterados de CT (43,5\%) e LDL-C $(29,9 \%)$ foram menores que os expressos em Santa Cruz do Sul. A frequência geral de dislipidemia foi de 54,3\%, bem como metade das crianças possuía pelo menos uma concentração anormal de lipídios (BIBILONI MDEL et al., 2015). Quando comparadas diferentes regiões de um mesmo país, observa-se que os níveis de lipídios podem variar conforme a região estudada (ZHU et al., 2012). Os valores de lipídios alterados podem estar associados à circunferência abdominal elevada proveniente de uma alimentação rica em carboidratos, bem como baixo nível de atividade física, além de histórico familiar de doenças cardiovasculares (SCB, 2013). 
Quanto ao HDL-C, os altos percentuais encontrados são preocupantes, pois há provas contundentes de que baixo nível de HDL-C no plasma é um importante fator de risco para doenças cardiovasculares (SCB, 2013). Os escolares de Santa Cruz do Sul apresentaram taxas mais elevadas de colesterol total, LDL-c e glicemia de jejum, sendo a combinação de elevado colesterol total e LDL-c associado a um maior risco de desenvolvimento de doença aterosclerótica precoce (SCB, 2013). Esses índices podem estar relacionados ao maior consumo de gordura (WU et al., 2014), açúcares (TE MORENGA et al., 2014), além da baixa ingestão de fibras (HARTLEY et al., 2016) e vegetais (HARTLEY et al., 2013).

Os resultados apresentados neste estudo, no que se refere aos hábitos alimentares, apontam que o consumo de frutas é maior entre os escolares de Chapecó. De acordo com os dados do IBGE (2010), este hábito é um indicador de alimentação saudável. Porém, esses mesmos escolares relataram maior consumo de doces e alimentos como pizzas/lasanhas. Sabendo que alterações no padrão alimentar influenciam o estado nutricional do indivíduo alterando seu perfil antropométrico e bioquímico, o consumo destes alimentos pode ser a causa de um elevado IMC e CC, além de níveis alterados de triglicerídeos nas crianças chapecoenses. Os escolares de Santa Cruz do Sul relataram maior consumo frequente/ diário de salgadinhos fritos e refrigerantes, o que pode ter relação com os níveis alterados de colesterol total, LDL-C e glicemia de jejum, verificados neste estudo.

Tal cenário é preocupante, tendo em vista que o consumo frequente destes alimentos constitui importante fator de risco para o desenvolvimento da obesidade e de diversas doenças crônicas não transmissíveis, tais como hipertensão arterial, doenças cardiovasculares e diversos tipos de câncer (PACÍFICO et al., 2011). Na Síria, um estudo comparativo com adolescentes, entre 15-18 anos, demonstrou que há diferenças significativas entre adolescentes, de ambos os sexos, no que se refere ao consumo de alimentos vegetais, carnes vermelhas, leites, fastfoods e alimentos açucarados. O estudo identificou que adolescentes do sexo masculino consomem mais fastfoods e refrigerantes $(52,4 \%$ vs. $43 \%)$, bem como possuem baixo consumo de frutas e verduras. Deste modo, esses dados mostram-se preocupantes, pois se estima que o alto consumo de fastfoods seja um fator de risco para obesidade (MUSAIGER; FALAN, 2014).

No que se refere aos hábitos alimentares, entendemos ser uma limitação do estudo, o fato de ser autorreferido, permitindo a omissão de informações importantes. Contudo, o presente estudo traz dados representativos de duas populações do sul do Brasil, aspecto que pode identificar se indicadores de saúde podem variar entre diferentes regiões, bem como descrever os hábitos comportamentais que têm influenciado a saúde de escolares brasileiros. Deste modo, é possível subsidiar melhor delineamento às ações de saúde que são direcionadas às crianças e adolescentes, considerando a realidade onde estes municípios estão inseridos.

\section{CONCLUSÃO}

Conclui-se que houve diferenças significativas no perfil nutricional, lipídico e hábitos alimentares de escolares de dois municípios do sul do Brasil, demonstrando que indicadores de saúde podem variar entre diferentes regiões. A prevalência de sobrepeso/ obesidade, CC elevada, triglicerídeos e HDL-C alterados foram maiores em Chapecó; colesterol total, LDL-C e glicemia alterados foram mais expressivas em Santa Cruz do Sul. Quanto aos hábitos alimentares, Chapecó apresentou maior consumo de frutas e doces, e Santa Cruz do Sul apresentou consumo elevado de feijão e arroz, refrigerantes e salgadinhos fritos. Deste modo, analisar a situação atual de saúde, bem como comparar diferentes regiões se faz necessário, para que futuras estratégias de saúde sejam mais efetivas, principalmente nos locais onde os índices mostram-se mais preocupantes.

\section{REFERÊNCIAS}

ABEP. Associação Brasileira de Empresas de Pesquisa. 2011. Disponível em: < http://www.abep.org/novo/Utils/ FileGenerate.ashx?id=197> . Acesso em: 03 ago. 2015.

BARROS, M. V. G.; NAHAS, M. V. Medidas da atividade física: teoria e aplicação em diversos grupos 
populacionais. Londrina: Midiograf, 2003.

BIBILONI MDEL, M.; SALAS, R.; NOVELO, H. I.; VILLARREAL, J. Z.; SUREDA, A.; TUR, J. A. Serum lipid levels and dyslipidaemia prevalence among 2-10 yearold Northern Mexican children. PLoS One, v. 10, n. 3, p. e0119877, 2015.

BLOCH, K. V. et al. ERICA: prevalências de hipertensão arterial e obesidade em adolescentes brasileiros. Revista de Saúde Pública, v. 50, n. supl. 1, p. 9s, 2016.

BURGOS, M. S.; REUTER, C. P.; BURGOS, L. T.; POHL, H. H.; PAULI, L. T. S.; HORTA, J. A.; RECKZIEGEL, M. B.; FRANKE, S. I. R.; PRÁ, D.; CAMARGO, M. Comparison analysis of blood pressure, obesity, and cardiorespiratory fitness in schoolchildren. Arquivos Brasileiros de Cardiologia, v. 94, n. 6, p. 739-744, 2010.

CABRERA, T. F. C.; CORREIA, I. F. L.; DOS SANTOS, D. O.; PACAGNELLI,F. L.; PRADO,M. T. A.; DA SILVA, T. D.; MONTEIRO,C. B. M.; FERNANI, D. C. G. L.Análise da prevalência de sobrepeso e obesidade e do nível de atividade física em crianças e adolescentes de uma cidade do sudoeste de São Paulo. Revista Brasileira de Crescimento e Desenvolvimento Humano, v. 24, n. 1, 67-72, 2014.

CDC/NCHS. Centers of Disease Control and Prevention. National Center of Health Statistics.Vital and Health Statistics, v. 11, n. 246, p. 01-192, 2002.

CHAPECÓ, Prefeitura Municipal. Disponibiliza informações gerais sobre o município. Disponível em: $<$ https://www.chapeco.sc.gov.br/>. Acesso em: $02 \mathrm{fev}$. 2017.

CONDE, W. L.; MONTEIRO, C. A. Body mass index cutoff points for evaluation of nutritional status in Brazilian children and adolescents. Journal of Pediatry, v. 82, n. 4, p. 266-272, 2006.

CORSO, A. C. T.; CALDEIRA G. V.; FIATES, G. M. R.; SCHMITZ, B. A. S.; RICARDO, G. D.; VASCONCELO, F. A. G. Fatores comportamentais associados ao sobrepeso e à obesidade em escolares do Estado de Santa Catarina. Revista Brasileira de Estudos Populacionais, v. 29, n.
1, p. 117-131, 2012.

CUREAU, F. G.; DUARTE, P. M.; SANTOS, D. L.; REICHERT, F. F.; ZANINI, R. R. Sobrepeso/obesidade em adolescentes de Santa Maria-RS: prevalência e fatores associados. Revista Brasileira de Cineantropometria \& Desempenho Humano, v. 14, n. 5, p. 517-526, 2012.

DREWNOWSKI, A.; MOUDON, A. V.; JIAO, J.; AGGARWAL, A.; CHARREIRE, H.; CHAIX, B. Food shopping behaviors and socioeconomic status influence obesity rates in Seattle and in Paris. International Journal of Obesity, v. 38, n. 2, p. 306-314, 2014.

FERRARI, G. L. M.; MATSUDO, V.; KATZMARZYK, P. T.; FISBERG, M. Prevalence and factors associated with body mass index in children aged 9-11 years. Jornal de Pediatria, 2017.

HARTLEY, L.; MAY, M. D.; LOVEMAN, E.; COLQUITT, J. L.; REES, K. Dietary fibre for the primary prevention of cardiovascular disease. The Cochrane Database of Systematic Reviews, n. 1, p. CD011472, 2016.

HARTLEY, L.; IGBINEDION, E.; HOLMES, J.; FLOWERS, N.; THOROGOOD, M.; ClARKE, A.; STRANGES, S.; HOOPER, L.; REES, K. Increased consumption of fruit and vegetables for the primary prevention of cardiovascular diseases. The Cochrane Database of Systematic Reviews, n. 6, p. CD009874, 2013.

IBGE. Instituto Brasileiro de Geografia e Estatística. Pesquisa de orçamentos familiares 2008-2009: antropometria e estado nutricional de crianças, adolescentes e adultos no Brasil. Rio de Janeiro: IBGE, 2010.

KATZMARZYK, P T.; BARREIRA, T. V.; BROYLES, S. T.; CHAPUT, J. P.;FOGELHOLM, M.; HU, G.; KURIYAN, R.;KURPAD, A.; LAMBERT, E. V.; MAHER, C.; MAIA, J.; MATSUDO, V.; OLDS, T.; ONYWERA, V.; SARMIENTO, O. L.; STANDAGE, M.; TREMBLAY, M. S.; TUDOR-LOCKE, C.; ZHAO, P.; CHURCH, T. S. Association between body mass index and body fat in 9-11-year-old children from countries spanning a range of human development. International Journal of Obesity Supplements, v. 5, 
n. suppl 2, p. S43-S46, 2015.

LIM, H.; YUE, H.; WANG, Y. Association betweenobesity and metabolic co-morbidities among children and adolescents in South Korea based on national data. BMC Public Health, v. 14, n. 279, p. 2-9, 2014.

LOHMAN, T. G. The use of skinfold to estimate body fatness on children and youth.Journal of Physical Education, Recreation \& Dance, v. 58, n. 9, p. 98-103, 1987.

MUSAIGER, A. O.; KALAN, F. Dietary habits and lifestyle among adolescents in Damascus, Syria. Annals of Agricultural and Environmental Medicine, v. 21, n. 2, p. 416-419, 2014.

PACÍFICO, L.; ANANIA, C.; MARTINO, F.; POGGIOGALLE, E.; CHIARELLI, F.; ARCA, M.; CHIESA, C. Management of metabolic syndrome in children and adolescents. Nutrition, Metabolism and Cardiovascular Diseases, v. 21, n. 6, p. 455-466, 2011.

PYSZ, M.; LESZCZYNSKA, T; CIESLIK, E; KOPEC, A. relationship between the intake energy and basic nutrients and the bmi values in group of children aged 10-12. Roczniki Państwowego Zakładu Higieny, v. 65, n. 4, p. 345-352, 2014.

SANTACRUZDO SUL, Prefeitura Municipal.Disponibiliza informações gerais sobre o município. Disponível em: $\quad<$ http://www.santacruz.rs.gov.br/municipio/ localizacao > . Acesso em: 02 fev. 2017.

SCB. Sociedade Brasileira de Cardiologia. V Diretriz Brasileira de Dislipidemias e Prevenção da Aterosclerose. Departamento de Aterosclerose da Sociedade Brasileira de Cardiologia. Arquivos Brasileiros de Cardiologia, v. 101, n. 4, 2013.

SLAUGHTER, M. H.; LOHMAN, T. G.; BOILEAU, R. A.; HORSWILL, C. A.; STILLMAN, R. J.; VAN LOAN, M. D.; BEMBEN, D. A. Skinfold equations for estimation of body fatness in children and youth. Human Biology, v. 60, n. 5, p. 709-723, 1988.

TAYLOR, R. W.; NEUTZLING, M. B.; ASSUNÇÃO, M. C.
F.; MALCON, M. C.; HALLAL; P. C.; MENEZES, A. M. B. Evaluation of waist circumference, waist-to-hip ratio, and the conicity index a screening tools for high trunk fat mass, as measured by dual-energy X-ray absorptiometry, in children aged 3-9 y. American Journal Clinical of Nutrition, v. 72, n. 2, p. 490-495, 2000.

TE MORENGA, L. A.; HOWATSON, A. J.; JONES, R. M.; MANN, J. Dietary sugars and cardiometabolic risk: systematic review and meta-analyses of randomized controlled trials of the effects on blood pressure and lipids. The American Journal of Clinical Nutrition, v. 100, n. 1, p. $65-79,2014$.

WANG, S.; YAN, D. V.; ZHENG, W.; ZHIYONG, Z.; JUN, $\mathrm{M}$. Trends in overweight and obesity among Chinese children of 7-18 years old during 1985-2014. Chinese journal of preventive medicine, v. 51, n. 4, p. 300-305, 2017.

WU, L.; MA, D.; WALTON-MOSS, B. HE, Z. Effects of low-fat diet on serum lipids in premenopausal and postmenopausal women: a meta-analysis of randomized controlled trials. Menopause, v. 21, n. 1, p. 89-99, 2014.

ZHU, J. F.; LIANG, L.; FU, J. F.; GONG, C. X.; XIONG, F.; LIU, G. L.; LUO, F. H.; CHEN, S. K. Survey on the levels of lipids in school-aged children of Beijing, Tianjin, Hangzhou, Shanghai, Chongqing and Nanning cities. Zhonghua Liu Xing Bing Xue Za Zhi, v. 33, n. 10, p. 1005-1009, 2012.

Recebido em: 2017-01-30 Aceito em: 2017-04-10 